\title{
Antecedentes sobre el desarrollo de la carcinología en Chile
}

\author{
Carlos G. Jara \\ Instituto de Zoología, Universidad Austral de Chile \\ Casilla 567, Valdivia, Chile
}

\begin{abstract}
RESUMEN. En Chile continental la utilización de los crustáceos se remonta al menos a 10.200 años atrás. Sin embargo, la descripción formal de los componentes de la carcinofauna chilena se inició hace alrededor de 200 años, reconociéndose a Juan I. Molina como el primer naturalista nacional y el primero en asignar nombres científicos a crustáceos de este país. El desarrollo de la carcinología en Chile es obra de un vasto conjunto de especialistas extranjeros y nacionales. Entre los extranjeros destacan viajeros como el inglés Charles R. Darwin, quien contribuyó al conocimiento de los cirripedios; el francés Alcide D’Orbigny, primero en describir decápodos fósiles de Chile; y el alemán Eduard Poeppig, a quien se debe la temprana descripción de decápodos marinos y dulceacuícolas. Entre las expediciones extranjeras destacan la del buque británico H.M.S."Challenger", la de la Universidad de Lund (Suecia) a Chile y la más reciente del B/I alemán "Victor Hensen" a Magallanes. Entre los naturalistas chilenos destacan Claudio Gay, Rodulfo A. Philippi, Carlos E. Porter y Nibaldo Bahamonde, todos ellos relacionados con el Museo Nacional de Historia Natural y el último reconocido como el más importante promotor del desarrollo contemporáneo de la carcinología en Chile. Hoy el conocimiento de la carcinofauna chilena de decápodos puede considerarse relativamente completo a nivel de taxonomía alfa, pero es preliminar en relación con la biología y ecología de la mayor parte de las especies.
\end{abstract}

Palabras claves: carcinología, historia, Chile.

\section{Background of carcinology progress in Chile}

\begin{abstract}
Although the utilization of crustaceans in continental Chile is at least 10,200 years old, the formal description of the components of the Chilean carcinofauna only began about 200 years ago; Juan I. Molina having being the first Chilean naturalist to scientifically name autochtonous crustacean species. Many foreign and national scientists have contributed, with their efforts, to the development of carcinology in Chile. Among the foreigners we find, Charles R. Darwin, who contributed to the knowledge of Chilean cirripeds; Alcide D'Orbigny, first in describing fossil decapods; and Eduard Poeppig, a German traveller who described marine and freshwater decapods. Among foreign expeditions that have contributed, that of H.M.S."Challenger", followed by the Lund University (Sweden) to Chile, and most recently that of the B/I "Victor Hensen" to Magallanes, are to be mentioned. Among Chilean naturalists, Claudio Gay, Rodulfo A. Philippi, Carlos E. Porter and Nibaldo Bahamonde, all of them related with the National Museum of Natural History (Chile), must be mentioned, especially the latter who is recognized as being the most influential promotor of carcinology in Chile during the second half of this century. At present, the inventory of the Chilean carcinofauna can be considered as complete, but little is still known about the biology and ecology of most of the species.
\end{abstract}

Key words: carcinology, history, Chile.

\section{INTRODUCCION}

Historia es, entre otras definiciones, un relato acerca de uno o más sucesos. Si dicho relato se atiene estrictamente a los hechos, sin interpretarlos, entonces adquiere el cariz de una crónica. La crónica es una narración objetiva pero, por lo mismo, carente de connotaciones valorativas de parte del narrador hacia los hechos que narra. 
La carcinología es una disciplina naturalística que, como las restantes disciplinas zoológicas, exige de quien la practica la decisión de dedicar tiempo y esfuerzo a conocer, admirar y querer a los crustáceos. El carcinológo es fundamentalmente un curioso ilustrado y por lo mismo se enamora de su quehacer y del de sus pares. $\mathrm{Si}$ un carcinológo se decide entonces a escribir algunas notas históricas acerca del quehacer que le entusiasma, dificilmente tales notas constituirán una crónica, porque por necesidad estarán teñidas por la emotividad y la subjetividad del relator. Por lo tanto, vemos el siguiente conjunto de notas como un aporte al eventual desarrollo de una Crónica de la Carcinología en Chile, lo que deseamos ocurra en un futuro no demasiado lejano.

\section{La carcinología en Chile prehispánico}

Aunque los fósiles de crustáceos son, aparentemente, abundantes en Chile, situación derivada del origen marino del territorio, muy pocos son los trabajos realizados referentes a restos paleontológicos de crustáceos, después de la obra de D’Orbigny (1842) y Philippi (1887). Solo destaca la síntesis de Camacho (1966), referente a los invertebrados fósiles sudamericanos, en la que se citan algunas especies de Chile. Posteriormente, el trabajo sobre Lebucarcinus de Bahamonde y Frassineti (1980) y los aportes de Luis Chirino referentes a la carcinofauna del terciario en Valdivia.

En relación a los primeros pobladores del territorio, la ausencia de lenguaje escrito entre los chilenos prehispánicos impidió la trasmisión hasta nosotros de un conocimiento tradicional, presumiblemente rico como todo conocimiento derivado de la recolección intencional de recursos. Sin embargo, excavaciones arqueológicas llevadas a cabo en las últimas décadas, han permitido vislumbrar que los crustáceos fueron utilizados por lo menos 10.200 años antes del presente (a.a.p.) por grupos humanos del denominado Período Arcaico Temprano de Los Vilos, en contextos culturales del Complejo Huentelauquén (Báez et al., 1993). Estos grupos consumieron preferentemente cangrejos. Otros grupos del Norte Grande integraron al camarón Cryphiops caementarius en su dieta y a Pagurus villosus en sus ritos. En sectores próximos a Iquique y Antofagasta esta situación continuó a través del Arcaico Medio (alrededor de 6.000 a.a.p.) y de todo el Período Agroalfarero. También se han encontrado restos de crustáceos, principalmente Homalaspis plana, que fueron consumidos por los primeros pobladores de isla Mocha (aproximadamente 3.300 a.a.p.) (Báez et al., 1994) y de Punta Curaumilla (entre 3.300 y 1200 a.a.p.). Sin lugar a dudas, los cangrejos inter e inframareales formaron también parte de la dieta habitual de pueblos recolectores del extremo sur de Chile (Chonos, Alacalufes y Onas).

El descubrimiento de la carcinofauna del Nuevo Mundo. Las expediciones extranjeras

Desde los lejanos tiempos del descubrimiento de América, la curiosidad científica se vió estimulada por la gran diversidad de organismos que se encontraron tan pronto como el Nuevo Mundo comenzó a ser incorporado a la trama de intereses de la civilización europeo-occidental. El interés que despertaron en Europa los especímenes animales y vegetales llevados desde "Las Indias" por los descubridores, y las notables descripciones que se hacían de la naturaleza en tierras americanas, debieron gravitar en la decisión de organizar las numerosas campañas dirigidas a explorar el Nuevo Continente, patrocinadas por las potencias comerciales del Viejo Mundo, para las cuales el conocimiento científico constituía un elemento fundamental del poder político.

Desde las expediciones pioneras llevadas a cabo por la corbeta "Coquille" (1822-1825), en las que Lesson no solo hizo mención de la taxonomía de las especies sino que dejó como legado interesantes observaciones biológicas, se sucedieron importantes expediciones. Ellas fueron encabezadas por la del H.M.S. "Beagle" (1831-1836), en la cual el naturalista inglés Charles Darwin (1809-1882) recogió los primeros materiales para su célebre obra sobre los cirripedios (Darwin 1851, 1854). Poco después, entre 1827 y 1829, el alemán Eduard Poeppig viajó por Chile, recolectando especímenes zoológicos y botánicos, incluyendo crustáceos. A continuación del "Beagle" visitaron aguas chilenas las expediciones de la "Bonite" (1836-37), de la fragata "Venus", de las corbetas "Astrolabe" y "Zelèe" (1837-1840), la Expedición Exploratoria de los Estados Unidos (1838-42), el Viaje por la América Meridional de Alcide D'Orbigny (184244), de la fragata austríaca "Novara" (1857-59), de la fragata italiana "Magenta" (1865-68), del "Nassau" (1866-69), del buque inglés H.M.S. "Challenger" (1872-76), del "Blake" al Golfo de México (1877-79) y al Caribe (1878-79), del "Alert" (187980), de la corbeta italiana "Vettor Pisani" (188285), la Misión Científica al Cabo de Hornos (188283), los viajes del "Albatross" y la Expedición Hamburguesa a Magallanes (1892-93). Fruto de 
ellas, la información acerca de la diversidad de la fauna marina del extremo sur del Continente Americano aumentó significativamente. De esta primera época de descubrimientos naturalísticos data la descripción de componentes notables de la carcinofauna chilena, tales como Pinnaxodes chilensis (Milne Edwards, 1837), Homalaspis plana (Milne Edwards, 1834) y Parastacus pugnax (Poeppig, 1835), entre otros.

Más tarde, nuevas expediciones se agregaron a las anteriores, entre ellas la Expedición Saint George al Pacífico (1924-25), la Expedición Zoológica Noruega a las Galápagos (1925), las exploraciones del "Eagle" y del "Ara" (1921-28), del "Alva" (1935), la Expedición TempletonCrocker (1936), y las diversas investigaciones realizadas por Allan Hancock, tanto en el Pacífico como en el Atlántico, a bordo del "Velero III" y "Velero IV" (1931-35). En los informes de todas ellas se encuentran referencias acerca de especies de crustáceos presentes en aguas jurisdiccionales de Chile.

La Expedición de la Universidad de Lund a Chile (Expedición Lund, 1948-49), dirigida por los Profs. Hans Brattström y Erick Dahl, produjo la fuente de información más importante sobre crustáceos decápodos chilenos publicadas en este siglo. La importancia de las publicaciones dedicadas a crustáceos radica, además, en la reconocida autoridad académica de sus autores, Holthuis (1952) (Decapoda Macrura); Haig (1955) (Decapoda Anomura); Garth (1957) (Decapoda Brachyura); Menzies (1962) (Isopoda); Holmquist (1957) (Mysidacea); Dahl (1954) (Stomatopoda y Mystacocarida); Nilsson-Cantell (1957) (Cirripedia Thoracica), y Boschma (1959) (Cirripedia Ascothoracica), como en la extensa revisión bibliográfica que aportan. Uno de los méritos más apreciables de estas publicaciones es haber reunido, ordenado y examinado en forma crítica, toda la información disponible hasta ese entonces sobre taxonomía y distribución geográfica de las especies. La falta de esta información habia sido percibida por los investigadores escandinavos durante su permanencia en Chile. Por este motivo, estas publicaciones fueron y son de enorme valor para quienes se interesan por los crustáceos y han servido de punto de partida para las investigaciones científicas de varios carcinólogos chilenos contemporáneos. En la misma serie de publicaciones existen otros dos trabajos de gran interés para biogeógrafos y ecólogos y muy útiles para carcinólogos (Brattström y Johanssen, 1983;
Brattström, 1990). Así, el conjunto de los cincuenta informes de la Expedición Lund solo se compara en valor e importancia con la obra "Historia Física y Política de Chile" editada por Claudio Gay (1849), con la obra "Fauna Chilensis" de Ludwig Plate, quien visitó Chile entre 1893 y 1895 , y con el "Saggio sulla storia naturale del Chili" del Abate Juan Ignacio Molina (1810), todas ellas clásicas en la literatura científica.

Los informes de la Expedición Lund sobre crustáceos decápodos reseñan 41 especies de macruros, de las cuales cuatro probablemente no pertenecen a la fauna chilena y que fueron incluidas debido a etiquetación errónea; entre éstas se encuentran Alpheus dentipes Guérin, Hippolyte coerulescens (Fabricius), Ibacus peroni Leach y Thalassina chilensis Steenstrup y Lütken. Se citan 48 especies de anomuros a las que podrían agregarse 3 especies problemáticas, i.e., Calcinus chilensis (Milne Edwards), Clibanarius aequabilis (Dana) e Isocheles aequimanus Dana; y 101 especies de braquiuros, de las cuales 27 son dudosas para Chile dada la procedencia del material. Como conclusiones importantes, se propone un esquema de distribución geográfica de las especies de decápodos que amerita ser conocido y considerado. Se constata que la mayor parte de las recolecciones de material chileno en aguas profundas había sido, hasta ese entonces (1949), hecha por el "Challenger". Se predice que nuevas expediciones en estas áreas del Pacífico Sudeste podrían revelar la existencia de numerosas especies nuevas, lo cual se ha confirmado en años ulteriores. La Expedición Lund fue quizás la primera en informar sobre hábitos y hábitat de especies de crustáceos que viven en Chile, comprobándose que el déficit de información sobre estos aspectos era muy grande. Esta situación ha cambiado poco, a pesar de los esfuerzos desplegados en las décadas siguientes por conocer la biología o algunos rasgos ecológicos de las especies de hábito intermareal o de especies de importancia económica.

Más recientemente, otras expediciones extranjeras hicieron aportes relativos especialmente a material zooplanctónico. Entre ellas se cuentan la “Tahiti-Nui II", de la "Downwind", los cruceros del "Bondy" y la "Expedición Antártica Soviética", todas en 1958; la del "Step I" (1960) y los cruceros del B/I "Eltanin” (1962-1963).

La última gran expedición oceanográfica que recolectó material carcinológico en el sistema de fosas Chile-Perú, en la Fosa Milne Edwards, fue la del buque norteamericano de investigación "Antonn 
Brunn”, que entre octubre y noviembre de 1965, bajo la dirección de Robert J. Menzies, hizo rastreos y dragados frente a la costa occidental de América del Sur. John Garth y Janet Haig prepararon una publicación sobre los decápodos anomuros y braquiuros recolectados allí. Posteriormente, en 1969, el R/V “Thomas Washington” efectuó cruceros frente a las costas chilenas, y durante 1974, a bordo del R/V “Alexander Agassiz”, se llevó a cabo la "Expedición Krill" en la que participaron destacados investigadores chilenos.

La expedición europea más reciente ha sido la campaña al estrecho de Magallanes del buque alemán B/I "Victor Hensen" (1994), bajo la dirección de Wolf Arntz. Entre los resultados de esta Expedición se encuentran seis artículos que amplian y completan el conocimiento de los crustáceos del extremo sur de América (Arntz y Gorny, 1996).

El descubrimiento de la carcinofauna del Nuevo Mundo. El aporte de los chilenos

El primer naturalista chileno es el Abate Juan Ignacio Molina (1740-1829). Nacido en Guaraculén, en las cercanías de Talca, perteneció a la Compañía de Jesús. En 1768 debió exiliarse, cuando apenas contaba con veintiocho años. Los integrantes de su Orden, por decreto del rey Carlos V de España, fueron expulsados del territorio chileno. En Bolonia, Italia, escribió anónimamente su "Compendio della storia geografica, naturale e civile del regno del Cile" (1788). En 1782 apareció su inmortal obra "Saggio sulla storia naturale del Chili", convirtiéndose así en el primer naturalista de este país, con ideas muy avanzadas para su época. En esa y otras obras sucesivas describió la naturaleza chilena, reseñando una serie de animales que ulteriormente resultaron ser especies nuevas para la ciencia. En concreto, en la segunda edición del "Saggio..." (1810), Molina cita 16 especies de crustáceos decápodos. Algunas de ellas no han sido aún reconocidas por investigadores extranjeros, dado que las características específicas y genéricas indicadas son insuficientes o confusas. Esta dificultad es mayor para los especialistas que no han visitado Chile, pero parece poco importante para quienes han venido a trabajar en terreno.

Lo siguiente ilustra el comentario del párrafo precedente. Entre las 13 especies de decápodos marinos descritas por Molina se encuentra Cancer santolla. Para cualquier chileno resulta claro, por el solo nombre, que se trata de la "centolla", que aunque parecida a las jaibas no es un decápodo braquiuro sino un anomuro. La autoría del nombre asignado por Molina no fué reconocida por más de un siglo, a pesar de la insistencia de R.A. Philippi. Conocida también como "barómetro araucano", el caparazón seco de centolla se ha empleado tradicionalmente en la zona de los canales australes para predecir el tiempo atmosférico, probablemente porque absorbe con rapidez la humedad ambiental y cambia de color. Igualmente, figuran en el Saggio algunas jaibas del género Cancer, que fueron luego reconocidas por Philippi $(1867,1894)$. Garth (1957) en el Apéndice B del informe sobre los Decápodos Braquiuros de la Expedición Lund, hace un interesante comentario acerca de los nombres que aparecen en la segunda edición de la obra de Molina (1810). No es este el lugar ni el momento para comentarlos. Sin embargo, desde nuestro punto de vista, la opinión sostenida por Garth en el punto 5 del apéndice es insostenible. Allí se afirma (sic) "Información suplementaria concerniente a Cancer setosus Molina, 1872 (1782?), indica que no es $C$. polyodon Poeppig, como lo sugirieron Philippi (1894) y Rathbun (1910), ni ninguna otra especie de Cancer, sino un cangrejo májido, posiblemente Libinia rostrata Bell, de Perú”. En nuestra opinión, Molina no pudo haber tenido en sus manos esta especie pero sí a Eurypodius latreillei, que se encuentra frecuentemente en Chiloé y canales australes.

Un caso muy especial es el del camarón de río del norte de Chile, común también al Perú, cuyo límite austral es el Río Maipo. Se le conoce actualmente como Cryphiops caementarius, aludiendo su nombre específico al hábito de excavar galerías en terrenos arcillosos anegados. El nombre caementarius es atribuido a Molina pero es probable que él se haya referido más bien a alguno de nuestros Parastacidae, ya que Parastacus pugnax, por ejemplo, es extraordinariamente abundante en las vegas de las vecindades de Talca y de Guaraculén, su tierra natal, y también en los alrededores de Bucalemu, donde los jesuítas tuvieron un convento. No se debe olvidar que las descripciones, hechas por Molina, se basaron en sus recuerdos y en algunos apuntes que un amigo suyo le llevó a Bolonia, durante el exilio.

Otra obra de conjunto sobre Chile es la de Alonso de Ovalle (1601-1651), autor de la "Histórica relación del reino de Chile", no solo estudió la historia civil del país sino también sus "producciones naturales". Esta obra, al igual que la de Molina, ha sido traducida a varios idiomas.

Muchos años transcurrieron hasta producirse la independencia política de Chile en 1810. En el intertanto, diversos viajeros extranjeros exploraron nuestro suelo y según Diego Barros Arana "habían 
dado a conocer sus riquezas en obras de verdadero mérito. Bastaría recordar los trabajos de Feuilléé i Frezier, "..que fueron reimpresos i traducidos a otros idiomas i hasta hoi se consultan con interés."

Durante el gobierno de José Miguel Carrera (1812-14), junto con la creación de la Biblioteca Nacional y del Instituto Nacional, se consultó la fundación del Museo Nacional cuyo primer Director debió ser Manuel de Salas. Pocas noticias se tienen sobre lo que ocurrió entonces pero se sucedieron varios directores que aparentemente no tuvieron la idoneidad deseada.

En la década de 1820, el Gobierno de Chile concluyó que las publicaciones de Molina, Ovalle y otros no habían logrado una difusión importante, eran muy incompletas y no daban, por tanto, una buena idea de la configuración del país ni de sus producciones. Por eso, el 14 de septiembre de 1830 se firmó el contrato entre el Gobierno de Chile y Claudio Gay (1800-1873), naturalista francés, quien se comprometió a realizar un catastro de los bienes naturales del país. Al decir de los historiadores, Claudio Gay "no tenía ni la ciencia, ni la fama de un verdadero sabio" para ejecutar la obra deseada y fundar además un Gabinete de Ciencias Naturales, el que habría dado origen al actual Museo Nacional de Historia Natural. Para asegurar el cumplimiento del contrato por parte de Claudio Gay, éste debió dejar en depósito su biblioteca, sus colecciones de Historia Natural y sus dibujos en casa de Francisco García Huidobro. Todos estos efectos deberían pasar a poder del Estado y ser depositados en la Biblioteca Nacional, en caso que, a juicio de la Comisión Supervisora, Claudio Gay no hubiere presentado resultados satisfactorios de sus trabajos después de seis meses, contados desde la fecha del contrato.

Manuel Gandarillas expresó en "El Araucano" No 3, del 3 de octubre de 1830. "Esta capital se adornará con un Gabinete de Historia Natural, a cuya vista nacerá en nuestros jóvenes la afición a la ciencia que recrea con utilidad al género humano i que produce ideas sublimes. Los extranjeros que lo visiten tendrán qué admirar, los sabios qué aprender i los manufactureros donde encontrar muestras de los materiales de sus establecimientos, clasificados i expresados con la nomenclatura técnica i su correspondiente vulgar".

Claudio Gay superó con creces las espectativas que se cifraron en él y al cabo de 40 años de trabajo produjo su monumental obra en varios volúmenes: ocho de historia, ocho de botánica, ocho de zoología, dos de documentos y dos Atlas en colores que aún causan admiración en quienes los consultan, por la belleza de sus láminas. Entre los tomos de Zoología, el volumen 3 está consagrado a los crustáceos y allí figuran 79 especies de Decapoda (49 braquiuros, 20 anomuros y 10 macruros) de las cuales seis están dibujadas en el Atlas. Las descripciones están en latín y a continuación traducidas y ampliadas al castellano. Sin embargo, muchas veces las versiones en latín y castellano se contradicen en los detalles, por lo que los científicos de la época recomendaban utilizar sólo las descripciones en latín. En ocasiones, según sus críticos, las descripciones son de tal manera oscuras o están tan equivocadas en los detalles, que aún los naturalistas de profesión no pueden formarse una idea cabal del objeto a que aluden. Algunas descripciones específicas reseñan los caracteres comunes a todas las especies del mismo género, de tal modo que es difícil encontrar los rasgos diagnósticos de cada una de ellas. Ocurre también que una especie aparece descrita en dos oportunidades bajo diferentes nombres; eventualmente, una descripción corresponde al macho y la otra a la hembra. Además se constata, según los críticos, una falta de precisión en la referencia a las localidades de recolección del material, i.e., "Chile", "costas de Chile", etc.

Resulta fácil criticar pero es difícil construir. Quien examine la obra en conjunto podrá aquilatar y reconocer el esfuerzo desplegado para llevarla a cabo y como ella corresponde al resultado de la estrecha colaboración entre selectos científicos de esa época. Guillermo Feliú Cruz escribió de Gay, en 1965, "Cuarenta años consagró Claudio Gay al servicio de Chile. La "Historia Física y Política de Chile" dió a conocer al país en el mundo europeo y fue la base del monumento a la gloria del sabio francés. Chile agradecido lo hizo su hijo adoptivo por una ley especial y le dió una pensión generosa que le permitió vivir con decoro...". Así, Claudio Gay devino el más señalado de los chilenos que han comprometido su esfuerzo en dar a conocer las producciones del país.

De Gay dijo Darwin: "celoso y hábil naturalista, ocupado entonces en estudiar todas las ramas de la historia natural del Reino de Chile". (Darwin, Journal of Researches, capítulo 12, citado por Diego Barros Arana). Chile lo distinguió como Ciudadano Honorario, título que fue su mayor orgullo y en cada volumen de la obra se lee "Claudio Gay, ciudadano chileno." 
Sería una omisión imperdonable no recordar aquí la obra de Rodulfo Armando Philippi (18081904), distinguido naturalista alemán, nacionalizado chileno, de fama mundial especialmente por sus trabajos malacológicos. Fué contratado para dirigir el Museo Nacional de Historia Natural y dar clases en el Instituto Nacional y en la Universidad de Chile. Marcó una huella indeleble por su sabiduría y por los viajes que realizó por diferentes partes del país, sobresaliendo su "Viaje al desierto de Atacama" realizado en 1854 y publicado en 1860, obra en la cual se encuentra información sobre crustáceos fósiles. Al parecer Philippi tenía en preparación un libro sobre crustáceos decápodos, el que indudablemente no alcanzó a terminar. En poder de la familia Philippi se encuentran láminas en colores y también algunas descripciones, inéditas. Algunas de estas láminas se encuentran depositadas en el Museo de la Universidad Austral de Chile, en Valdivia.

En 1897, un joven naturalista llamado Carlos Emilio Porter Mosso (1867-1942) fundó en Valparaíso la Revista Chilena de Historia Natural, modesto órgano divulgatorio científico que es uno de los más antiguos de habla española. Por ella fue conocido Chile en los círculos científicos del exterior. Representaba a la Ciencia chilena y ha sido su mejor exponente en lo que se refiere a Historia Natural. La iniciativa de Porter, en el campo zoológico, fue fecunda. Su mayor interés fueron los artrópodos, especialmente insectos y crustáceos decápodos.

Porter fué Director del Museo de Historia Natural de Valparaíso. Es probable que allí mantuviera una colección de crustáceos decápodos, peces y ejemplares de otros grupos taxonómicos que formaban parte de las colecciones del Museo, las cuales fueron destruidas por el terremoto del 16 de agosto de 1906. Se desempeñó además como Jefe de la Sección Invertebrados del Museo Nacional de Historia Natural, en Santiago, y fue profesor en la Escuela de Altos Estudios que funcionó en el mismo Museo, donde dictó, entre otras asignaturas, un curso sobre zoogeografía de invertebrados.

Por su erudición pronto es reconocido como "el sabio Porter". Su labor científica fue fructífera, resultado de una labor infatigable, con mucho esfuerzo, tenacidad, perseverancia y amor a la Ciencia. Fue un gran ejemplo para las generaciones de la época en que vivió y para las que le siguieron. Más de 400 publicaciones atestiguan esta afirmación. Entre ellas son numerosas las que se refieren a crustáceos decápodos. El profesor Francisco Riveros Zúñiga, primer zoólogo de la Estación de Biología Marina de Montemar, envió a John Garth, de la Allan Hancock Foundation, California (USA), una lista de las publicaciones carcinológicas de Porter, la que constituye el Apéndice 1 del volumen Braquiuros de la Expedición de la Universidad de Lund a Chile.

Carlos Porter publicó un gran número de notas ampliando el rango geográfico de varias de las especies de crustáceos decápodos conocidas en esa época. Algunas de las especies registradas por Porter no se han vuelto a registrar en aguas chilenas, como sucede con algunos grápsidos de origen tropical que seguramente llegaron a Chile, en estado adulto, juvenil o larvario, con algún evento de El Niño-Oscilación del Sur, sin que esos colonizadores lograran afincarse en nuestras costas.

A comienzos del presente siglo las publicaciones más recientes sobre crustáceos decápodos del Pacífico meridional occidental eran las de Mary Rathbun, distinguida carcinóloga estadounidense. Rathbun (1910) publicó el "Catálogo sobre los Crustáceos Podoftalmos del Perú" y otros sobre diversos grupos de Brachyura de América, algunos de los cuales tuvieron caracter monográfico. Carlos Porter mantuvo con Rathbun una nutrida correspondencia, al igual que con numerosos carcinólogos y naturalistas norteamericanos y europeos, correspondencia que sumada a las publicaciones que recibía regularmente por canje de la Revista Chilena de Historia Natural, lo mantenían al día en cuanto a la literatura científica de la época. Además, con frecuencia, enviaba a sus colegas extranjeros ejemplares para su identificación o para confirmar la identificación que él había hecho. Dichos ejemplares quedaban en poder de cada uno de ellos, mientras la información recibida a cambio se constituía en ayuda inestimable para Porter en sus tareas en los Museos así como en la Escuela de Agronomía de la Universidad de Chile, donde mantuvo colecciones de decápodos.

Porter tuvo oportunidad de describir por lo menos dos especies de decápodos muy importantes para el país y para la ciencia; i.e., Cervimunida johni y Paromola rathbuni. Al mismo tiempo, algunos decápodos descritos en el extranjero recibían su nombre, como por ejemplo Cancer porteri Rathbun.

Porter ha sido un ejemplo señero para los naturalistas chilenos. Recibió distinciones que nadie, en este campo, ha superado. Se le nombró profesor Honorario, doctor Honoris Causa, Miembro Correspondiente de varias Sociedades Científicas, y recibió numerosas medallas y condecoraciones de 
varios países, como muestra de reconocimiento. Falleció en Santiago el 13 de diciembre de 1942.

Trás la desaparición de Porter la comunidad naturalística nacional consideró que el estudio de los crustáceos decápodos estaba prácticamente agotado. Parecía poco juicioso dedicar tiempo a nuevas investigaciones sobre estos animales, sobre todo si se consideraba que había en Chile otros grupos zoológicos de los cuales nada se sabía en esa fecha. La dinámica del desarrollo comercial y académico futuro demostraría que dicha presunción era equivocada. Nibaldo Bahamonde retomaría el grupo y pronto se sumaron a las investigaciones sobre crustáceos, María Teresa López, Elda Fagetti, Tarcisio Antezana, Italo Campodonico, Leonardo Guzmán, Carlos Viviani, Guacolda Atria, Verónica Alegría, Gabriel Henríquez, Alberto Carvacho, Patricio Arana, Sergio Palma, Matilde López, Marco A. Retamal, y muchos más. Ninguno de ellos necesita ya presentación, pues han dedicado gran parte de su tiempo a estudios carcinológicos, resolviendo competentemente problemas concretos. Otros investigadores más jóvenes y entusiastas han colaborado con nuevos bríos en el curso de las últimas décadas: Eric Rudolph (Parastacidae), Exequiel González (Amphipoda), Pablo Schmiede (Porcellanidae), Pedro Báez y Enrique Dupré (Palinuridae), Carlos Jara (Aeglidae), Jaime Meruane, Miguel Rivera (Palaemonidae), y muchos otros que sería largo enumerar. Los conocimientos se han multiplicado en cuanto a ciclos biológicos, ecología, fisiología, sistemática y taxonomía, pesquerías, entre otros tópicos. Pero quedan grandes vacíos por llenar en cuanto a genética, etología, evolución, biogeografía, cultivos, aprovechamiento integral de los recursos, reconocimiento de la fauna de aguas profundas, etc. El problema mayor es contar con personal competente, con un nivel científico adecuado que le permita ser reconocido internacionalmente, que tenga a su disposición laboratorios y bibliotecas en concordancia con sus necesidades, o por lo menos contar con contactos que le permitan suplir las carencias. Junto a ello, es necesario mantener grupos que apoyen eficientemente el trabajo del investigador, incorporando continuamente a los jóvenes.

Desarrollo de las pesquerías de crustáceos en Chile y estudio de las poblaciones explotadas

Es ampliamente reconocida la apetencia de los chilenos por el consumo de mariscos, especialmente de moluscos y crustáceos. Este hecho motivó que los pescadores artesanales se dedicaran desde la época colonial a la extracción de recursos disponibles en la región costera. No obstante, no fue sino después de la Segunda Guerra Mundial cuando se inició la captura de crustáceos de aguas profundas, dando origen a su explotación comercial. Este desarrollo fue posible con la llegada al país de buques provistos de la tecnología apropiada.

Después de 1945 los estudios sobre recursos pesqueros actuales y potenciales fueron principalmente descriptivos, para ampliar los limitados antecedentes disponibles. Ellos cubrieron aspectos taxonómicos, distribucionales y de biología básica. Con el paso del tiempo las investigaciones se fueron ampliando espacialmente y se enfocaron aspectos cada vez más específicos. Preludio de ellos fueron los trabajos pioneros, conducidos por Elda Fagetti en la Estación de Biología Marina de Montemar, sobre desarrollo larvario en el laboratorio de cangrejos intermareales del área de Valparaíso. En esa escuela se formaron destacados investigadores, como Tarcisio Antezana, Italo Campodonico y Leonardo Guzmán.

Un hito muy importante en el desarrollo de estudios aplicados sobre la fauna carcinológica chilena lo constituyó la creación del Instituto de Fomento Pesquero (IFOP) en 1964, apoyado inicialmente por la Organización de las Naciones Unidas para la Agricultura y la Alimentación (FAO). El naciente Instituto desarrolló las primeras exploraciones sistemáticas de crustáceos presentes en la plataforma continental y en el talud superior. En ellas participaron expertos extranjeros, como Michael Mistakidis y Allan Hancock, junto a profesionales chilenos, los que más tarde debieron hacerse cargo de la dirección de las investigaciones al finalizar el convenio con FAO.

En los años transcurridos desde su creación, el IFOP ha realizado una cantidad de estudios e investigaciones que han permitido disponer de una visión general e integral de los crustáceos en aguas jurisdiccionales chilenas, tanto de las especies explotadas como de las potencialmente explotables. Parte de estas labores fueron conducidas por Gabriel Henríquez, Roberto Bahamonde, Teresa Peñailillo y, más recientemente, por Rubén Roa.

Contemporáneamente,en diversas universidades se comenzaron a formar grupos de trabajo que se orientaron a la investigación de los recursos explotados. Tal es el caso de la actual Escuela de Ciencias del Mar de la Universidad Católica de Valparaíso, con una larga tradición de trabajos 
biológico-pesqueros principalmente sobre la langosta de Juan Fernández (Jasus frontalis), langostino colorado (Pleuroncodes monodon), langostino amarillo (Cervimunida johni), camarón de roca (Rhynchocinetes typus) y camarón nailon (Heterocarpus reedi). Estas investigaciones han sido lideradas por Patricio Arana y secundadas por Patricio Pavez y Sergio Palma.

No menos importante ha sido la labor desarrollada en el Instituto de la Patagonia, actualmente dependiente de la Universidad de Magallanes, en Punta Arenas, donde se han destacado Italo Campodonico y Leonardo Guzmán, quienes trabajaron en equipo e incluyeron esporádicamente a otros investigadores. Hicieron importantes publicaciones sobre biología de la centolla (Lithodes santolla) y del centollón (Paralomis granulosa).

Más recientemente la Universidad Católica del Norte (Sede Coquimbo) ha logrado estructurar un prometedor equipo de investigadores, orientados fundamentalmente al cultivo de crustáceos. $\mathrm{Ha}$ desarrollado tecnologías para el cultivo masivo de camarón de río del norte (Cryphiops caementarius) y de especies foráneas que podrían ser introducidas para su crianza en ambientes controlados, con fines comerciales. Entre esas especies se cuentan camarones peneidos (Penaeus spp.) y especies de agua dulce como Macrobrachium spp. y la langosta australiana Cherax quadricarinatus. En estas labores se empeñan Jaime Meruane, Enrique Dupré y Miguel Rivera.

Otros centros universitarios también han contribuido al desarrollo de la carcinología, a través de estudios directos o indirectos de especies de interés comercial. En ellos se han destacado investigadores como Juan C. Castilla (Pontificia Universidad Católica), Ernst Kilian (Universidad Austral de Chile), Héctor Andrade, Raúl Soto (Universidad Arturo Prat), Víctor A. Gallardo (Universidad de Concepción), y muchos otros. A esta lista se debe agregar un sinnúmero de contribuciones esporádicas hechas por tesistas e investigadores que en forma puntual $\mathrm{u}$ ocasional han aportado al conocimiento biológico-pesquero de los recursos carcinológicos presentes en aguas chilenas.

\section{Problemas y tendencias actuales}

Hoy ya no es necesario justificar, ante los organismos estatales y privados del país, el intercambio de investigadores entre diversas partes del globo y solo es necesario alentarlo y apoyar la obtención de financiamiento para mantenerlo. Sin embargo, es necesario promover, justificar y financiar expediciones de exploración a la fauna marina y límnica.

Por otra parte, la calidad y cantidad de medios de comunicación ha hecho que las distancias se acorten. Así, cada vez es más fácil el contacto entre investigadores, tanto dentro del país como entre países. Los métodos para obtención y análisis de información se han automatizado, pero sigue siendo imprescindible el investigador para planificar el trabajo, interpretar los resultados y controlar la automatización. Hoy más que ayer, el trabajo en equipo es indispensable.

Hasta hace un par de decenios carecíamos en Chile de bibliotecas científicas especializadas en el área marina. Hoy ellas existen pero aún son bastante incompletas. Este último problema se ha solucionado en los últimos años gracias a la automatización de bibliotecas y centros de documentación, y al intercambio interbibliotecario. Las facilidades que ofrece la comunicación electrónica y el uso prefencial del inglés como idioma de la ciencia ha hecho más expedita la obtención e intercambio de información. Aún así, las labores carcinológicas básicas, entre ellas la identificación de especies, continúa siendo una tarea en la que se empeña un reducidísimo contigente de especialistas que han cultivado al menos dos virtudes, la paciencia y la constancia.

A pesar de las dificultades, se ha logrado estructurar un archivo científico que ha acumulado diagnosis, descripciones y rasgos biológicos fundamentales relativos a los organismos que habitan la región del Pacífico Sureste y nuestro país. Ejemplo de ello han sido las publicaciones CORFO/ IFOP sobre crustáceos, las sinopsis publicadas por FAO, los catálogos publicados por Bahamonde y López (1963), Henríquez y Bahamonde (1976), y Retamal (1981), entre otros. En este último se registran 227 especies e ilustran 208 de ellas, constituyéndose en la obra contemporánea de consulta más recurrida sobre los crustáceos decápodos de Chile. Toda esta información se ha incrementado paulatinamente gracias al trabajo tesonero de otros naturalistas latinoamericanos, principalmente peruanos y argentinos, que en número creciente pero aún insuficiente, han descrito las novedades que han encontrado. Muchos de ellos han contribuido así, indirectamente, al mejor conocimiento carcinológico de Chile.

El cuadro histórico de la carcinología en Chile se completa en la última década con la incorporación de un contingente numeroso de estudiantes y académicos a la tarea de conocer 
detalles de todo tipo acerca de la carcinofauna, tanto marina como dulceacuícola. Gran parte de este conjunto de investigadores se ha reunido en torno a la Sociedad Chilena de Carcinología, fundada en mayo de 1994, en gran medida como resultado del empuje y entusiasmo de Ingo Wehrtmann, investigador alemán que se avecindó en Valdivia por cinco años (1991-1996), a través del "Servicio Alemán de Intercambio Académico" (DAAD), en el Instituto de Zoología de la Universidad Austral de Chile. Wehrtmann estableció una amplia plataforma de trabajo en torno al desarrollo larvario de decápodos, particularmente Caridea y en esta empresa involucró directamente a estudiantes de Biología Marina y de otras procedencias. Sin embargo, el principal motor de la carcinología en esta segunda mitad del siglo XX ha sido la labor incansable del profesor Nibaldo Bahamonde, cuyo estimulante consejo y admirable capacidad de asombro ha catalizado la labor de todos los que han continuado las investigaciones en torno a los crustáceos de Chile. Su meritorio aporte se sintetiza no sólo en las tesis sobre temas carcinológicos que ha guiado (9 de un total de 34 ), sus numerosas publicaciones sobre el mismo tema (50 de un total cercano a las 160), así como libros, textos de estudio y cursos impartidos. Su participación en congresos, seminarios y reuniones científicas sobre el tema, junto a su condición de miembro del comité editor de las principales revistas científicas chilenas, le ha permitido resaltar la importancia que reviste para el país el estudio científico de los crustáceos, su rol en los ecosistemas acuáticos, su valor para la economía nacional y la necesidad de investigación científica para la evaluación y prognosis de estos recursos. Por su prolongada y fructífera labor en favor de las Ciencias del Mar en Chile fué distinguido, en 1983, como Miembro de Número de la Academia de Ciencias del Instituto de Chile y en 1996 con el Premio Nacional de Ciencias, máxima distinción que otorga el país a sus académicos destacados, situación que honra y eleva la Carcinología chilena a un alto sitial.

Hoy la Sociedad Chilena de Carcinología cuenta con 65 socios, de los cuales más de dos tercios eran estudiantes en el momento de su afiliación. Ello muestra el enorme interés que los crustáceos despiertan en las generaciones más jóvenes de científicos, augurando un promisorio futuro para el desarrollo de esta disciplina en Chile. Será tarea de los carcinólogos de la nueva generación recoger estos apuntes, ampliarlos y escribir la Historia de la
Carcinología Chilena, en el marco riguroso de un trabajo científico. Por ahora, sean estas notas un estímulo y una guía para esa tarea.

\section{AGRADECIMIENTOS}

El autor agradece especialmente al Profesor Nibaldo Bahamonde el haberle proporcionado el manuscrito de la conferencia "Breve retrospectiva sobre la carcinología en Chile", que dictó en el Curso de postgrado "Biología de Crustáceos Decápodos en Chile", en la Universidad Austral de Chile, en octubre de 1995, que sirvió de base para estructurar este artículo. Expresa además su reconocimiento por la valiosa y desinteresada colaboración de árbitros y editores, quienes contribuyeron anónimamente no sólo con indicaciones y sugerencias para mejorar el texto, sino también con materiales para enriquecerlo. Quedo en deuda con todos ellos, dejando en claro, sin embargo, que la responsabilidad que pueda haber por omisiones involuntarias y eventuales injusticias históricas cometidas en esta recopilación, es del autor. Se agradece a la Sra. Patricia Araya G., la traducción y redacción del Abstract.

\section{REFERENCIAS}

Arntz, W. y M. Gorny. 1996. Cruise report of the Joint Chilean German-Italian Magellan "Victor Hensen" Campaign in 1994. Berichte zur Polarforschung, 190: 1-113.

Báez, P., D. Quiroz y D. Jackson. 1993. Crustáceos en contextos arqueológicos. Museos, Coordinación nacional de Museos DIBAM, 17: 12-15.

Báez, P., D. Jackson y D. Quiroz. 1994. Crustáceos en la pesca prehispánica de Chile: Antecedentes arquelógicos. Chile Pesquero, 81: 48-52.

Bahamonde, N. 1948. Observaciones biológicas sobre la langosta de Juan Fernández (Jasus frontalis H. Milne Edwards). Rev. Biol. Mar., Valparaíso, 1(2): 90-102.

Bahamonde, N. y M.T. López. 1963. Decápodos de aguas continentales en Chile, Invest. zool. chil., 10: $123-149$.

Bahamonde, N. y D. Frassinetti. 1980. Lebucarcinus n. gen. del Mioceno de Chile (Crustacea, Decapoda, Brachyura). Boln. Mus. Nac. Hist. Nat. Chile, 37: 275-279. 
Boschma, H. 1959. The Crustacea Rhizocephala of Chile. Reports of the Lund University Chile Expedition 1948-49, \#37. Lunds Universitet Arsskrift. N.F. Avd. 2, Bd. 56, 3: 20 pp.

Brattström, H. y A. Johanssen. 1983. Ecological and regional zoogepgraphy of the marine benthic fauna of Chile. Report $\mathrm{N}^{\circ} 49$ of the Lund University Chile Expedition 1948-49, Sarsia, 68(4): 289-339.

Brattström, H. 1990. Intertidal ecology of the northernmost part of the Chilean archipelago. Report $\mathrm{N}^{\circ} 50$ of the Lund University Chile Expedition. Sarsia, 75: 107-160.

Camacho, H. 1966. Invertebrados fósiles. Editorial Universitaria de Buenos Aires, EUDEBA, 707 pp.

Dahl, E. 1954. Stomatopoda. Reports of the Lund University Chile Expedition 1948-49, \#15. Lunds Universitet Arsskrift. N.F. Avd. 2, Bd. 49, 17: 12 pp.

Darwin, C.R. 1851. A Monograph on the Subclass Cirripedia 1. Lepadidae, London, 1851.

Darwin, C.R. 1854. A Monograph on the Subclass Cirripedia 2. The Balanidae, Verrucidae, etc., London, 1854.

D'Orbigny, A. 1842. Coquilles et les échinodermes fossiles recueillés en Colombie, par M. Boussingnault. In: Voyage dans l'Amerique Méridionales, Paléont., 66.

Garth, J. 1957. The Crustacea Decapoda Brachyura of Chile.- Reports of the Lund University Chile Expedition 1948-49, \#29. Lunds Universitet Arsskrift. N.F. Avd. 2, Bd. 53, 7: 127 pp.

Haig, J. 1955. The Crustacea Anomura of Chile.- Reports of the Lund University Chile Expedition 1948-49, \#20. Lunds Universitet Arsskrift. N.F. Avd. 2, Bd. 51, 12: 68 pp.

Henríquez, G. y N. Bahamonde. 1976. Clave de identificación de jaibas frecuentes en las pescas comerciales de Chile (Crustacea, Decapoda, Brachyura). Ser. cient., Inst. fom. pesq., Santiago, 21: 1-73.

Holmquist, Ch. 1957. Mysidacea of Chile. Reports of the Lund University Chile Expedition 1948-49, \#28. Lunds Universitet Arsskrift, N.F. Avd. 2, Bd. 53, 6: $53 \mathrm{pp}$.

Recibido el 03 de marzo 1997.

Aceptado el 04 de julio 1997.
Holthuis, L.B. 1952. The Crustacea Decapoda Macrura of Chile.- Reports of the Lund University Chile Expedition 1948-49, \#5. Lunds Universitet Arsskrift.N.F.Avd. 2, Bd. 47, 10: $110 \mathrm{pp}$.

Menzies, R.J. 1962. The Zoogeography, ecology, and systematics of the chilean marine Isopods.- Reports of the Lund University Chile Expedition 1948-49, \#42. Lunds Universitet Arsskrift. N.F. Avd. 2, Bd. 57, 11: 162 pp.

Milne Edwards, H. 1834. Histoire naturelle des Crustacés, comprenant l'anatomie, la physiologie et la classification de ces animaux, Paris, Vol. 1, 468 pp.

Milne Edwards, H. 1837. Histoire naturelle des Crustacés, comprenant l'anatomie, la physiologie et la classification de ces animaux, Paris, Vol. 2: 532 pp. .

Nilsson-Cantell, C-A. 1957. Thoracic Cirripeds from Chile.- Reports of the Lund University Chile Expedition 1948-49, \#31. Lunds Unisitet Arsskrift, N.F. Avd. 2, Bd. 53, 9: 25 pp.

Philippi, R.A. 1860. Reise durch die wueste Atacama auf Befehl der Chilenischen Regierung im Sommer 1853-54 unternommen und beschrieben, 27 pls. Halle.ix +192 pp.

Philippi, R.A. 1867. Comentario crítico sobre los animales descritos por Molina. Anales de la Universidad de Chile, 29: 775 y siguientes.

Philippi, R.A. 1887. Los fósiles terciarios i cuartarios de Chile, 58 láms. Imprenta Brockhaus, Leipzig, 256 pp.

Philippi, R.A. 1894. Dos palabras sobre la sinonimia de los Crustáceos Decápodos Braquiuros o jaivas de Chile. Anales de la Universidad de Chile, 87: 369-376.

Poeppig, E. 1835-6. Reise in Chile, Peru und auf dem Amazonenstrome, während der Jahre 1827-1832. Vol. 1, Leipzig.

Rathbun, M.J. 1910. The stalk-eyed Crustacea of Peru and the adyacent coast. Proc. U.S. Nat. Mus., 38: 531-620.

Retamal, M. 1981. Catálogo Ilustrado de los Crustáceos Decápodos de Chile. Gayana (zool.), 44: 1-110. 